\title{
Vasopressin to attenuate pulmonary hypertension and improve systemic blood pressure after correction of obstructed total anomalous pulmonary venous return
}

\author{
Mark A. Scheurer, MD, Scott M. Bradley, MD, and Andrew M. Atz, MD, Charleston, SC
}

$\mathrm{P}$ ulmonary hypertension remains a major contributor to morbidity in the postoperative course of patients after successful surgical correction of obstructed total anomalous pulmonary venous return (TAPVR). Additionally, the postcardiotomy syndrome of low systemic vascular resistance and low cardiac output often necessitates aggressive vasopressor and inotropic support. Vasopressin has been prospectively shown in both adults and children to decrease the need for vasoactive medications in the postcardiotomy syndrome. In animal models of hypoxic pulmonary constriction, vasopressin has been shown to have properties of pulmonary vasodilation as well as systemic vasoconstriction. We hypothesized that vasopressin might decrease pulmonary vascular resistance (PVR) and thus decrease the need for vasoactive medications after surgical correction of TAPVR.

\section{Clinical Summaries}

PATIENT 1. A 36-week gestation 2.3-kg female infant with obstructed supracardiac TAPVR underwent complete repair on the second day after birth. After weaning from cardiopulmonary bypass, pulmonary arterial pressure (PAP) increased to suprasystemic levels. Nitric oxide (NO) was initiated at $40 \mathrm{ppm}$. On arrival in the pediatric cardiac intensive care unit, the patient had a PAP of $51 / 21 \mathrm{~mm} \mathrm{Hg}(65 \%$ systemic) and was receiving dopamine (10 $\left.\mu \mathrm{g} \cdot \mathrm{kg}^{-1} \cdot \min ^{-1}\right)$, epinephrine $\left(0.03 \mu \mathrm{g} \cdot \mathrm{kg}^{-1} \cdot \min ^{-1}\right)$, and milrinone $\left(0.5 \mu \mathrm{g} \cdot \mathrm{kg}^{-1} \cdot \mathrm{min}^{-1}\right)$. Management included hyperventilation, hyperoxygenation, paralysis, and sedation. Epinephrine was gradually increased to $0.1 \mu \mathrm{g} \cdot \mathrm{kg}^{-1} \cdot \min ^{-1}$ to treat systemic hypotension. PAP concomitantly increased to near systemic levels and was treated by increasing NO to $60 \mathrm{ppm}$. At 20 hours after the patient's arrival in the unit, vasopressin was initiated at 0.0003 units $\cdot \mathrm{kg}^{-1} \cdot \mathrm{min}^{-1}$ (Figure 1). Within 1 hour, PAP had decreased to $55 \%$ of systemic levels, and systemic blood pressure had increased from 55 to $85 \mathrm{~mm} \mathrm{Hg}$. An echocardiogram on postoperative day 1 showed a widely patent pulmonary venous anastomosis site. Vasopressin was titrated upward to a maximal dose of 0.0012 units $\cdot \mathrm{kg}^{-1} \cdot \min ^{-1}$. Epinephrine was rapidly

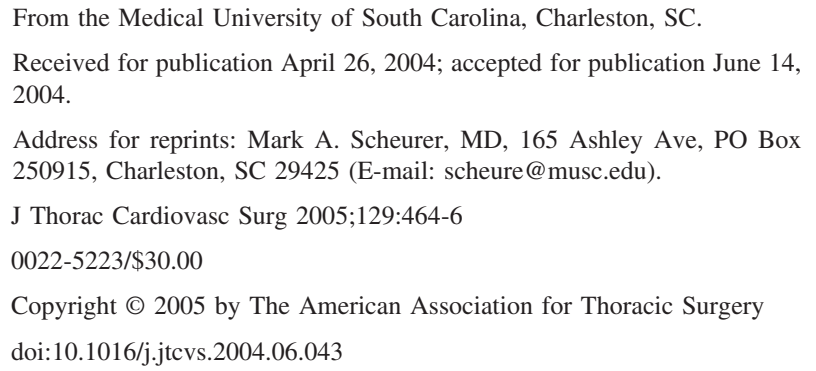

tapered and stopped within 24 hours of initiation of vasopressin. PAP remained between $55 \%$ and $75 \%$ of systemic during this period. After 82 hours, vasopressin was discontinued because of hyponatremia (117 mmol/L). No elevation in PAP was noted after discontinuation of vasopressin. Sodium levels returned to normal within the next 24 hours. NO was stopped within 24 hours after vasopressin discontinuation with no rebound pulmonary hypertension. The child was extubated on postoperative day 10 and was discharged on postoperative day 22. At 13.5 months of age, the child is thriving with no evidence of recurrent pulmonary venous obstruction or pulmonary hypertension.

PATIENT 2. A term 3.4-kg male infant with obstructed infracardiac TAPVR underwent surgical correction on the first day after birth. The patient was successfully weaned from cardiopulmonary bypass. PAP was initially systemic, and NO at 20 ppm was initiated. On arrival in the pediatric cardiac intensive care unit, the patient had a PAP of 58/26 mm $\mathrm{Hg}$ (80\% systemic) and was receiving dopamine $\left(10 \mu \mathrm{g} \cdot \mathrm{kg}^{-1} \cdot \mathrm{min}^{-1}\right)$ and milrinone $(0.5 \mu \mathrm{g}$ $\left.\cdot \mathrm{kg}^{-1} \cdot \min ^{-1}\right)$. Management included hyperventilation, hyperoxygenation, paralysis, and sedation. Epinephrine was initiated at $0.02 \mu \mathrm{g} \cdot \mathrm{kg}^{-1} \cdot \mathrm{min}^{-1}$ for systemic hypotension. As PAP rose to near systemic levels 2 hours later, vasopressin was initiated at 0.0003 units $\cdot \mathrm{kg}^{-1} \cdot \min ^{-1}$ and epinephrine was turned off. Within 1 hour, PAP had decreased to $60 \%$ of systemic and systemic blood pressure had stabilized. An echocardiogram on postoperative day 1 showed a widely patent pulmonary venous anastomosis site. Vasopressin was titrated upward to a maximal dose of 0.0012 units $\cdot \mathrm{kg}^{-1} \cdot \mathrm{min}^{-1}$. After 60 hours, vasopressin was discontinued because of hyponatremia $(119 \mathrm{mmol} / \mathrm{L})$. No elevation in PAP was noted after discontinuation of vasopressin. Sodium levels returned to normal within the next 36 hours. NO was stopped within 72 hours after vasopressin discontinuation with no rebound pulmonary hypertension. The child was extubated on postoperative day 8 and was discharged on postoperative 18 . At 13 months of age, the child is thriving with no evidence of recurrent pulmonary venous obstruction or pulmonary hypertension.

\section{Discussion}

Previous reports have shown that vasopressin is an effective agent in adults to decrease systemic vasopressor requirements with postcardiotomy syndrome. ${ }^{1}$ In children with congenital heart disease and postoperative vasodilatory shock, one case series similarly showed a decrease in inotropic scores immediately after initiation of continuous vasopressin. ${ }^{2}$ In that series, changes in PVR or PAP were not reported outcomes. In adult series, PAP during vasopressin infusion has variously been reported as being either unchanged or decreased, with no significant changes in PVR. ${ }^{1}$ A collection of animal experiments has shown that vasopressin selectively vaso- 

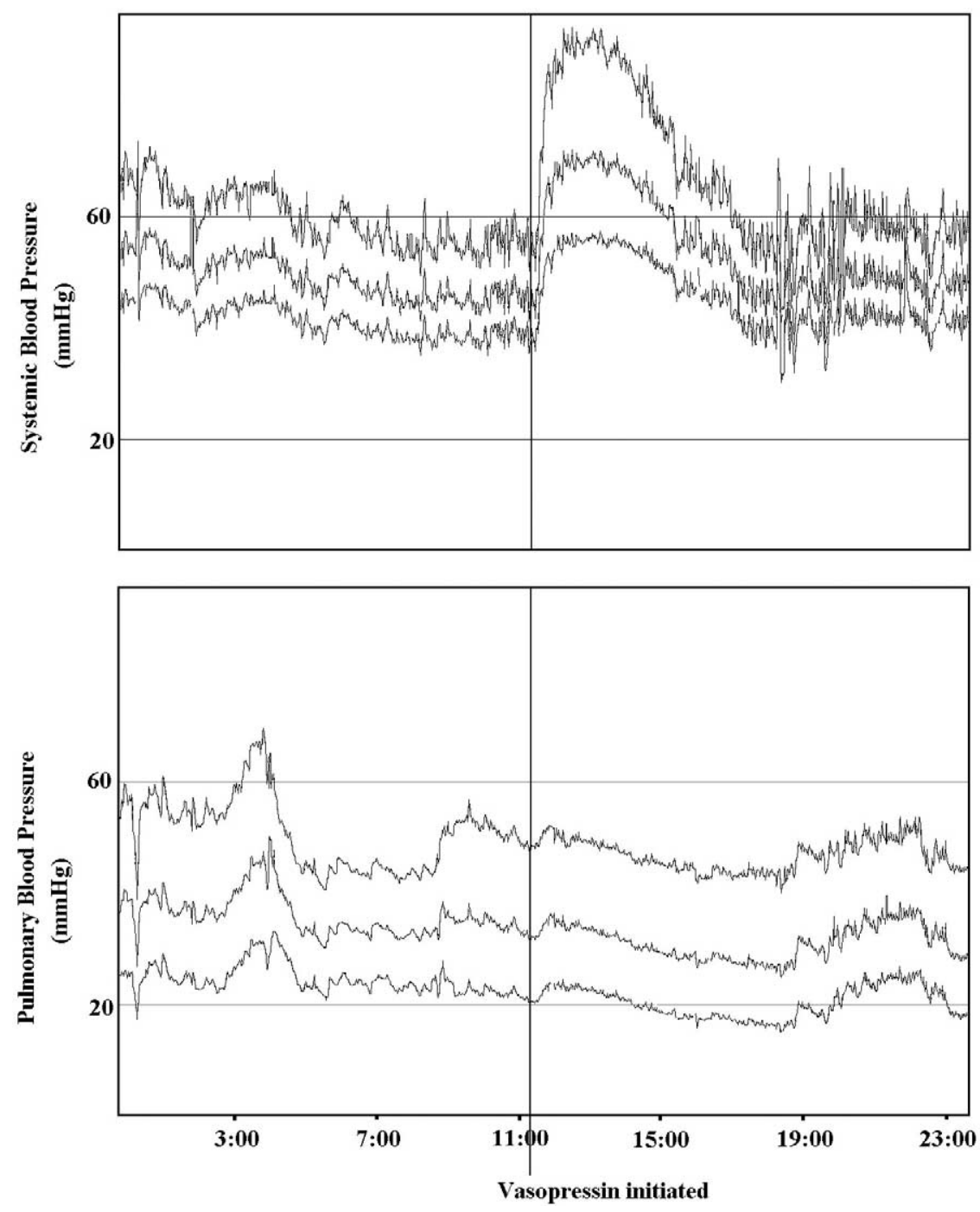

Figure 1. Continuous traces of systemic blood pressure (systolic, mean, and diastolic), and PAP (systolic, mean and diastolic) recorded every minute in patient 1 . Despite treatment with $\mathrm{NO}$ at $60 \mathrm{ppm}$, patient had persistently elevated PAP. Continuous infusion of vasopressin $(0.0003 \mathrm{units} /[\mathrm{kg} \cdot \mathrm{min}]$, initiated at vertical line) caused additional decrease in PAP and improved systemic blood pressure.

dilates pulmonary vasculature under hypoxic conditions through a $\mathrm{V}_{1}$ receptor-mediated release of NO.,4 Additionally, a model of chronic hypoxia suggests that the selective pulmonary vasodilatory action of vasopressin is enhanced through altered receptor-mediated processes during prolonged hypoxia. ${ }^{5}$

We hypothesized that in patients with high risk of vasodilatory shock, a history of a significant hypoxic insult, and coincident pulmonary hypertension (such as infants after repair of obstructed TAPVR) vasopressin would be an effective agent to decrease the need for vasopressor support and to ameliorate pulmonary hypertension. The 2 cases presented here demonstrate that the initiation of vasopressin can dramatically decrease PAP and increase systolic blood pressure, allowing the decrease of vasopressor infusions. Although no direct measurements of PVR or cardiac output were obtained during this study, left atrial pressures remained the same, and no increase in lactic acidosis ensued after the initiation of vasopressin. Most importantly, the decreases in PAP were noted during a period of otherwise maximized medical therapy for pulmonary hypertension.

The use of a vasopressin infusion does have limitations, such as the hyponatremia noted in these cases. These decreases in sodium were gradual and transient and did not result in clinical seizures. Continuous electroencephalographic monitoring was not performed, however, so the presence of subclinical seizures cannot be excluded.

We have found that vasopressin can have an important temporizing role in the control of pulmonary hypertension and systemic hypotension after obstructed TAPVR repair. Measurement of arginine vasopressin levels before and after initiation of vasopressin, as well as calculation of cardiac output and PVR, would be important in further prospective studies in such patients. Caution 
should be used when using vasopressin beyond 24 hours, because significant hyponatremia may ensue.

\section{References}

1. Morales DL, Gregg D, Helman DN, Williams MR, Naka Y, Landry DW, et al. Arginine vasopressin in the treatment of 50 patients with postcardiotomy vasodilatory shock. Ann Thorac Surg. 2000;69:102-6.

2. Rosenzweig EB, Starc TJ, Chen JM, Cullinane S, Timchak DM, Gersony WM, et al. Intravenous arginine-vasopressin in children with vasodilatory shock after cardiac surgery. Circulation. 1999;100(19 Suppl):II182-6.

3. Walker BR, Haynes JJr, Wang HL, Voelkel NF. Vasopressin-induced pulmonary vasodilation in rats. Am J Physiol. 1989;257(2 Pt 2):H415-22.

4. Evora PR, Pearson PJ, Schaff HV. Arginine vasopressin induces endothelium-dependent vasodilatation of the pulmonary artery: V1-receptormediated production of nitric oxide. Chest. 1993;103:1241-5.

5. Eichinger MR, Walker BR. Enhanced pulmonary arterial dilation to arginine vasopressin in chronically hypoxic rats. Am J Physiol. 1994; 267(6 Pt 2):H2413-9.

\title{
Extended pericardiotomy avoids cardiopulmonary bypass during bilateral sequential lung transplantation
}

\author{
Takahiro Oto, MD, Marc Rabinov, FRACS, Franklin Rosenfeldt, FRACS, and \\ Donald S. Esmore, FRACS Melbourne, Australia
}

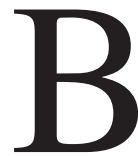

ilateral-sequential lung transplantation (BSLTx) through a clamshell thoracotomy has made the procedure of double-lung replacement possible without the use of cardiopulmonary bypass (CPB). ${ }^{1}$ Avoiding CPB has the potential advantages of reducing early allograft dysfunction $^{2}$ and coagulopathy, as well as lessening the amount of blood transfusion. ${ }^{3} \mathrm{CPB}$ is always required during lung transplantation for pulmonary hypertensive lung diseases or nonpulmonary hypertensive lung diseases with concomitant cardiac repair. However, $\mathrm{CPB}$ is also occasionally required during lung transplantation for inadequate gas exchange, right-heart dysfunction, or hemodynamic instability caused by mediastinal manipulation during single-lung ventilation. ${ }^{2}$ For patients with pulmonary fibrosis $(\mathrm{PF})$ undergoing BSLTx, adhesions and reduced size of the pleural space might necessitate forceful mediastinal manipulation to access the left hilum. This might cause severe hemodynamic compromise, despite inotropic support and inhaled nitric oxide. Unplanned CPB might be required, especially when the patients with $\mathrm{PF}$ have preexisting pulmonary hypertension ( $\mathrm{PH})$. To avoid hemodynamic instability during mediastinal manipulation, we postulated that extending the pericardiotomy might release the right

\footnotetext{
From the Department of Cardiothoracic Surgery, The Alfred Hospital, Monash University, Melbourne, Australia.

Received for publication June 16, 2004; revisions received June 24, 2004; accepted for publication June 30, 2004.

Address for reprints: Takahiro Oto, MD, Heart and Lung Transplant Unit, The Alfred Hospital, Commercial Road, Melbourne 3004, Australia (Email: takahirooto@aol.com).

J Thorac Cardiovasc Surg 2005;129:466-7

$0022-5223 / \$ 30.00$

Copyright $\odot 2005$ by The American Association for Thoracic Surgery

doi:10.1016/j.jtcvs.2004.06.045
}

heart from compression, improve cardiac function, and avoid the necessity of CPB. We have performed this procedure in 3 patients with moderate $\mathrm{PH}$ caused by $\mathrm{PF}$ and succeeded in all 3 in avoiding the unplanned use of CPB.

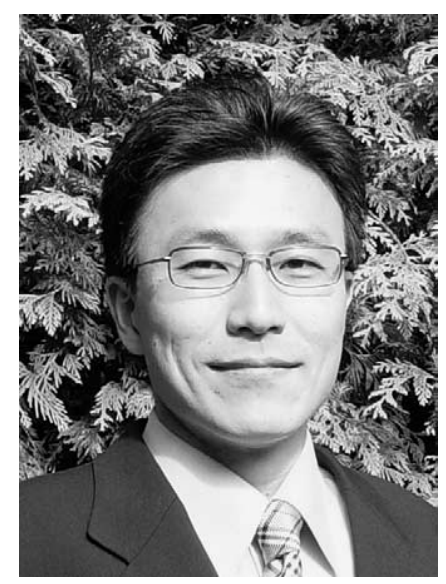

Dr Oto

\section{Clinical Summary}

Between March 1990 and May 2004, a total of 500 lung transplantations were performed at the Alfred Hospital, and of these, 239 were BSLTx procedures (Figure 1). Six of 21 patients with PF had severe preexisting $\mathrm{PH}$; that is, the ratio of pulmonary artery pressure (PAP) to systemic blood pressure (PAP/AoP) was more than 0.8 , and planned $\mathrm{CPB}$ was performed. For the 15 patients with moderate $\mathrm{PH}(\mathrm{PAP} / \mathrm{AoP}$ ratio of $<0.8), \mathrm{CPB}$ was on standby in the operating room and was initiated when needed. All 15 patients had an acceptable hemodynamic status approximately 10 minutes after clamping of the left pulmonary artery. All 10 patients with PAP/ AoP ratios of less than 0.5 did not require CPB to complete the procedure. For the 5 patients with $\mathrm{PAP} / \mathrm{AoP}$ ratios of between 0.5 and 0.8 (Table 1), severe hemodynamic instability resulted from mediastinal manipulation to access the left hilum and pulmonary ligament during the left-side procedure. Efforts made to avoid CPB included use of inotropic drug infusions (epinephrine or norepinephrine up to $2.5 \mu \mathrm{g} \cdot \mathrm{kg}^{-1} \cdot \mathrm{min}^{-1}$ ), vasodilators (sodium nitroprusside, nitroglycerin, or prostacyclin), and inhaled nitric oxide (40 ppm). Despite these treatments, PAP and central venous pressure increased with decreased $\mathrm{AoP}$ in all 5 patients, and initiation of $\mathrm{CPB}$ seemed to be unavoidable to complete the procedure. In the first 2 patients of this series, CPB was immediately initiated; however, in the last 3 patients, we elected to perform a longmidline pericardiotomy. The midline pericardiotomy extended to- 